\title{
Transient Type 1 Brugada Pattern without Ongoing Fever during COVID-19 Pneumonia
}

\author{
Maria Cristina Pasqualetto ${ }^{1}$, Andrea Corrado $^{2}$, Eleonora Secco ${ }^{1}$, Fabio Graceffa ${ }^{1}$, Fausto Rigo ${ }^{1}$ \\ ${ }^{1}$ Division of Cardiology, Dolo Hospital AULSS 3, Venice, Italy \\ 2 Division of Cardiology, Mestre Ospedale dell'Angelo AULSS 3, Venice, Italy
}

Received: 14/06/2020

Accepted: $15 / 06 / 2020$

Published: $19 / 06 / 2020$

How to cite this article: Pasqualetto MC, Corrado A, Secco E, Graceffa F, Rigo F. Transient type 1 brugada pattern without ongoing fever during COVID-19 pneumonia. EJCRIM 2020;7: doi:10.12890/2020_001808.

Conflicts of Interests: The Authors declare that there are no competing interests.

This article is licensed under a Commons Attribution Non-Commercial 4.0 License

\section{ABSTRACT}

Patients with COVID-19 pneumonia can develop heart problems and may also may be susceptible to proarrhythmia, virus-related issues such as fever, stress, electrolyte disturbance and adverse effects from the use of antiviral drugs. We report a transient Brugada-like ECG pattern without ongoing fever in a 57-year-old man, admitted with a diagnosis of COVID-19 pneumonia, who did not have syncope or a family history of sudden cardiac death.

\section{LEARNING POINTS}

- Patients with COVID-19 pneumonia can develop heart problems.

- A transient Brugada-like ECG pattern was observed in a non-febrile COVID-19 patient.

- SARS-CoV-2 may have a direct effect on myocardial ion channels.

\section{KEYWORDS}

Brugada pattern, COVID-19, fever, myocardial ion channels

\section{CASE DESCRIPTION}

We report a transient Brugada-like ECG pattern without ongoing fever in a 57-year-old man who was admitted to our hospital with a diagnosis of COVID-19 pneumonia. He had a 6-day history of fever and cough and was admitted to the emergency department (ED) because of worsening dyspnoea and chest pain. The nasopharyngeal swab was positive for COVID-19. At admission, the chest x-ray did not clearly show typical COVID-19 findings, which actually appeared 6 days later (Figs. 1 and 2). The patient did not have a family history of sudden death, syncope or cardiovascular disease, but did have a medical history of non-Hodgkin lymphoma.

A normal $\mathrm{PaO}_{2} / \mathrm{FiO}_{2}$ ratio was obtained with nasal cannula high flow oxygen therapy. High-sensitive troponin T and NT-proBNP were both normal. CRP was increased (122.5 mg/l at admission) with normal procalcitonin, and progressively decreased during hospitalization, becoming nearly normal at discharge (11.8 $\mathrm{mg} / \mathrm{l})$.

The ECG at admission to the ED was performed during fever $\left(38.5^{\circ} \mathrm{C}\right)$ and showed a right bundle branch block (Fig. 3) with diffuse low voltage. A routine ECG performed 2 days later showed a COVID-type Brugada pattern on V1 and V2 precordial leads (Fig. 4) without ongoing fever or other symptoms. A daily ECG was then performed, and to check QT interval duration during lopinavir/ritonavir and hydroxychloroquine therapy. Echocardiography performed according to our local protocol was normal. 


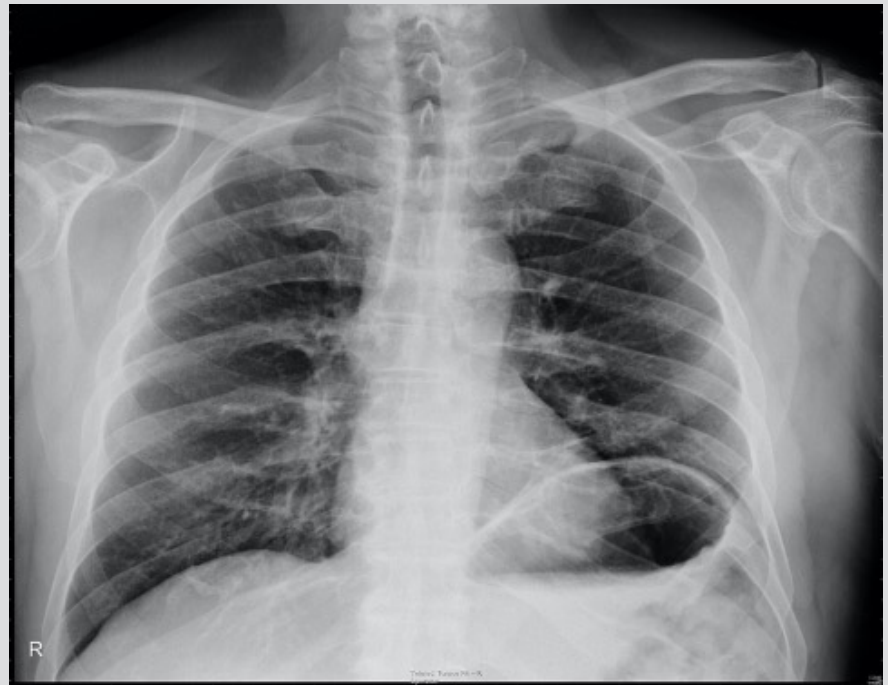

Figure 1. Chest $x$-ray at admission in the emergency department, without typical COVID-19 findings

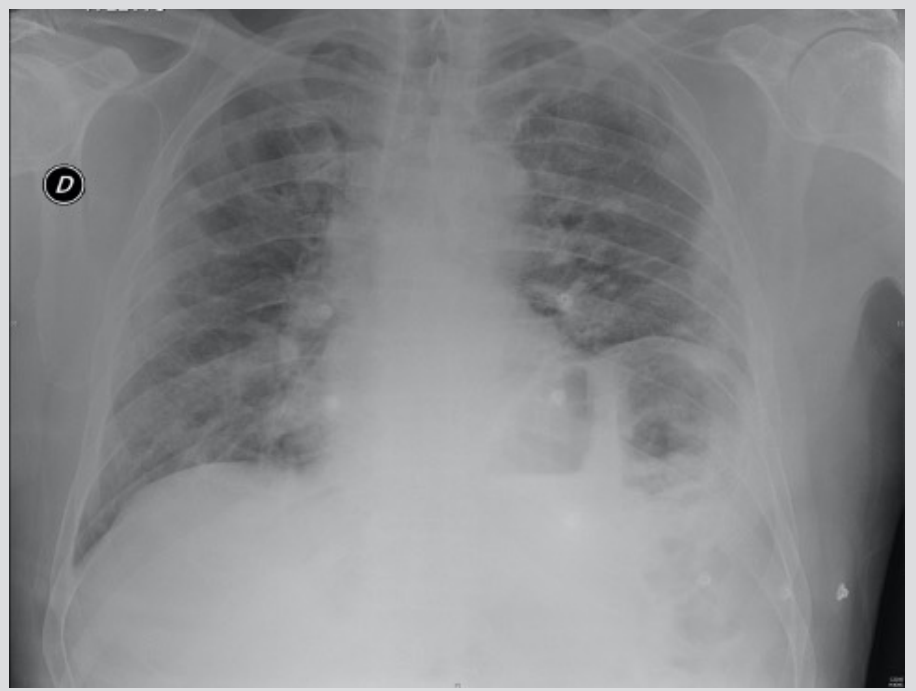

Figure 2. Chest $x$-ray 6 days later showing bilateral consolidation of the lungs

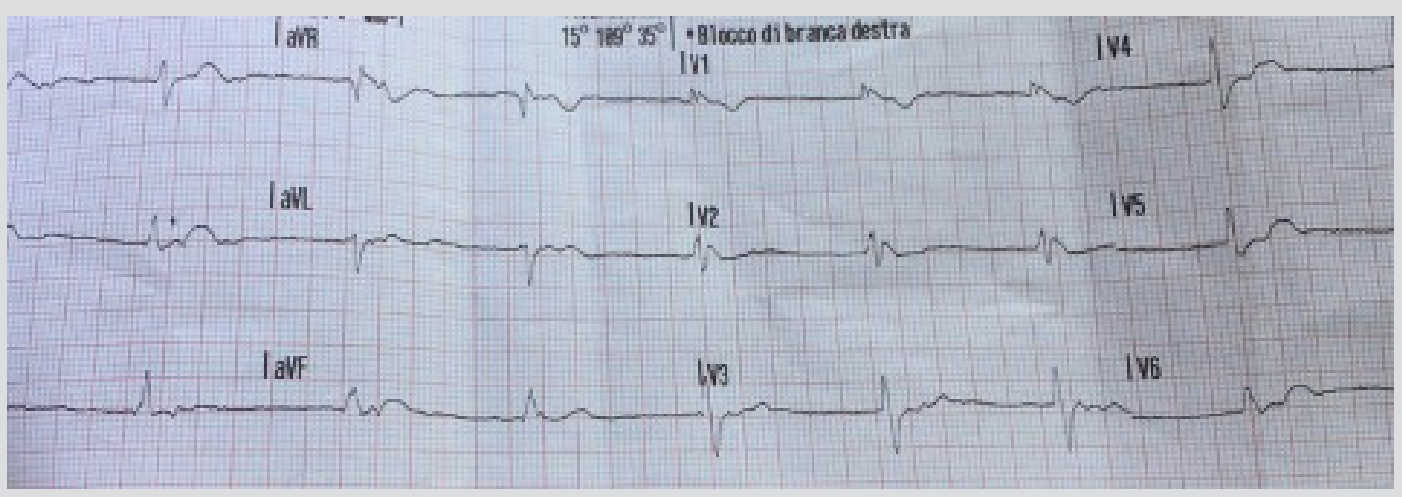

Figure 3. ECG at admission in the emergency department with fever

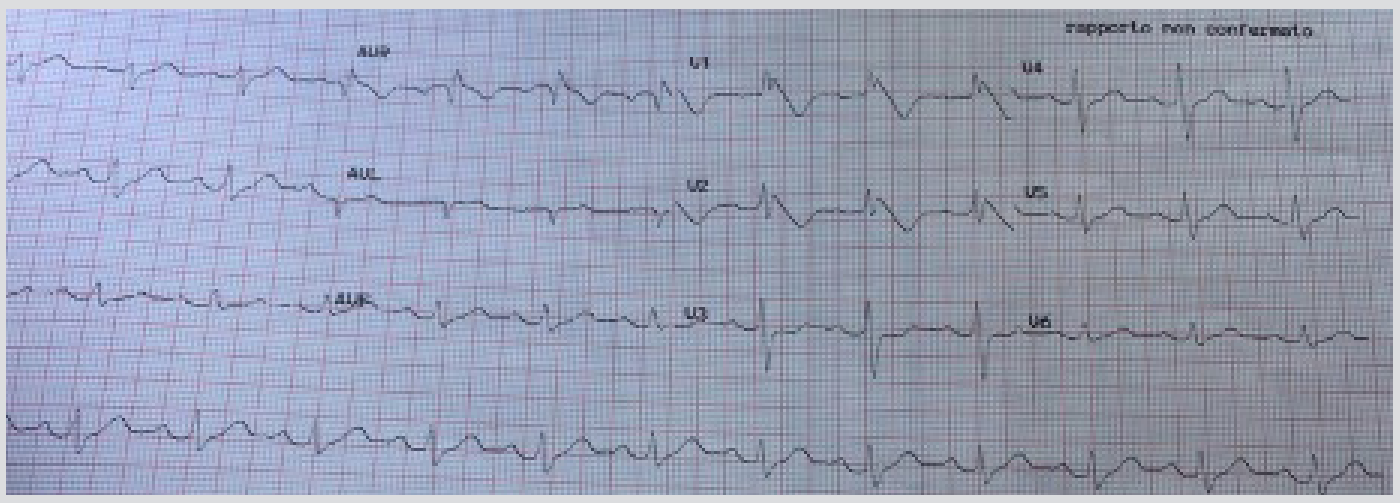

Figure 4. ECG without fever (type 1 Brugada pattern)

The patient had a good clinical course with rapid improvement, and ECG showed complete resolution of previous findings. He was discharged after a couple of weeks. Follow-up 1 month later showed complete normalization of ECG and chest x-ray findings, confirming resolution of the COVID-19 pneumonia (Fig. 5).

Given that the patient had no history of syncope/arrhythmias and had a COVID-19-related Brugada ECG pattern, clinical follow-up was mandatory even though the risk of major adverse cardiac events was low ${ }^{[1]}$. There is a well-known association between fever and a Brugada type I pattern which has been described extensively ${ }^{[2-4]}$, including in COVID-19 patients ${ }^{[5]}$. 


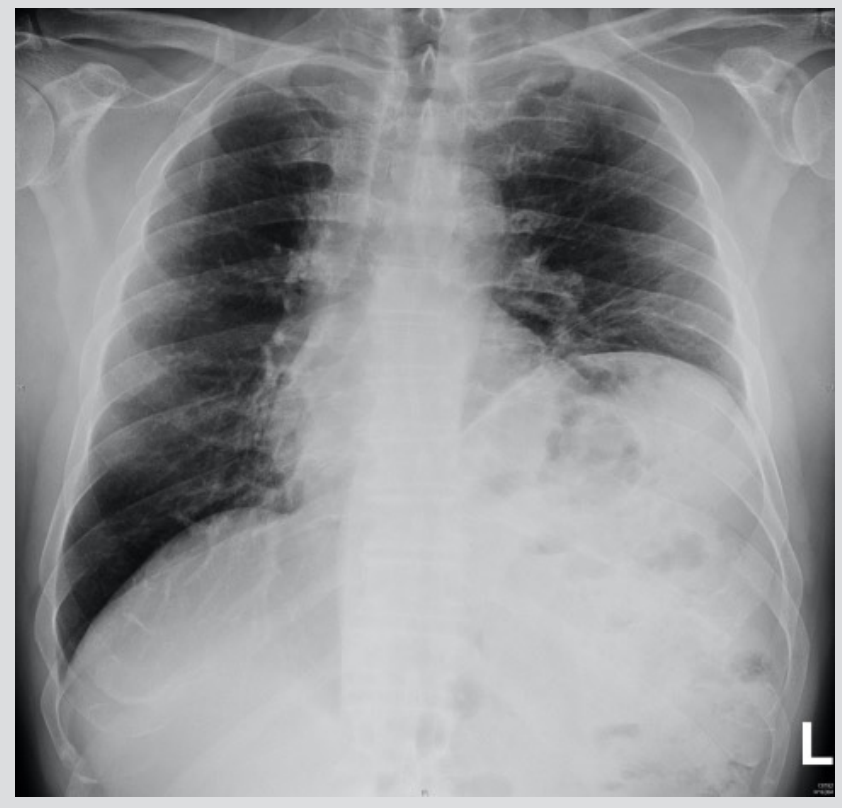

Figure 5. Chest $x$-ray 1 month after discharge showing resolution of COVID-19

pneumonia

This is the first case of a Brugada type 1 ECG pattern without ongoing fever in COVID-19 disease. Whether this ECG finding was preexisting or should be considered the Brugada phenotype in the clinical setting of COVID-19 infection is unclear.

\section{DISCUSSION}

The aetiology of cardiac injury seen in some COVID-19 patients seems to be multifactorial and involves direct viral myocardial damage, hypoxia, hypotension, enhanced inflammatory status, ACE2-receptor downregulation, drug toxicity, and endogenous catecholamine adrenergic status ${ }^{[6]}$.

Moreover, the Brugada phenotype is a clinical entity that is characterized by ECG patterns identical to those of Brugada syndrome but which arise from different underlying conditions such as metabolic abnormalities, myocardial ischaemia and mechanical compression ${ }^{[7]}$. Brugada phenotype patterns are classified the same as those of Brugada syndrome (i.e., types 1 and 2), but differ substantially regarding aetiology and prognosis.

There is currently no supporting evidence, but it is possible that the virus may interact directly with the myocardial ion channels and provoke ECG modifications typical of Brugada syndrome ${ }^{[8]}$, or the disturbance may be the result of other combined effects of the virus or of ongoing therapies.

Our case is important because the presence of the Brugada pattern, without ongoing fever, could confirm the hypothesis of a direct effect of the virus on myocardial ion channels. Investigation with an ajmaline test should be considered in the follow-up of COVID-19 patients who develop this ECG pattern, in order to determine its persistence as well as for a better definition of risk and prognosis.

\section{REFERENCES}

1. Al-Khatib SM, Stevenson WG, Ackerman MJ, Bryant WJ, Callans DJ, Curtis AB, et al. 2017 AHA/ACC/HRS guideline for management of patients with ventricular arrhythmias and the prevention of sudden cardiac death: a report of the American College of Cardiology/American Heart Association Task Force on Clinical Practice Guidelines and the Heart Rhythm Society. J Am Coll Cardiol 2018;72:e91-e220.

2. Mizusawa Y, Morita H, Adler A, Havakuk O, Thollet A, Maury P, et al. Prognostic significance of fever-induced Brugada syndrome. Heart Rhythm 2016;13:1515-1520.

3. Amin AS, Meregalli PG, Bardai A, Wilde AA, Tan HL. Fever increases the risk for cardiac arrest in the Brugada syndrome. Ann Intern Med 2008;3:216-218.

4. Barra S, Providencia R, Nascimento J. Fever outperforms flecainide test in the unmasking of type 1 Brugada syndrome electrocardiogram. EP Europace 2013;3:394.

5. Vidovich M. Transient Brugada-like ECG pattern in a patient with coronavirus disease 2019 (COVID-19). JACC Case Rep 2020 Apr 16. doi: 10.1016/j.jaccas.2020.04.007 [Epub ahead of print].

6. Kochi AN, Tagliari AP, Forleo GB, Fassini GM, Tondo C. Cardiac and arrhythmic complications in patients with COVID-19. J Cardiovasc Electrophysiol 2020;31(5):1003-1008.

7. de Oliveira Neto NR, de Oliveira WS, Mastrocola F, Sacilotto L. Brugada phenocopy: mechanisms, diagnosis, and implications. J Electrocardiol 2019;55:45-50.

8. Sorgente A, Capulzini L, Brugada P. The known into the unknown: Brugada syndrome and COVID-19. JACC Case Rep 2020 Apr 16. doi: 10.1016/j.jaccas.2020.04.006 [Epub ahead of print]. 